\title{
Heavy-Light Mesons Scattering from Nucleons: Quark-Gluon and Meson Exchanges
}

\author{
C.E. Fontoura \\ Instituto de Física Teórica, Universidade Estadual Paulista \\ Rua Dr. Bento Teobaldo Ferraz, 271 - Bloco II 01140-070 São Paulo, SP
}

\begin{abstract}
We investigate the scattering of heavy-light $K$ and $D$ mesons by nucleons at low energies. The short-distance part of the interaction is described by quark-gluon interchange and the longdistance part is described by a one-meson-exchange model that includes the contributions of vector $(\rho, \omega)$ and scalar $(\sigma)$ mesons. The microscopic quark model incorporates a confining Coulomb potential extracted from lattice QCD simulations and a transverse hyperfine interaction consistent with a finite gluon propagator in the infrared. The derived effective meson-nucleon potential is used in a Lippmann-Schwinger equation to obtain s-wave phase shifts. Our final aim is to set up a theoretical framework that can be extended to finite temperatures and baryon densities.
\end{abstract}

Keywords: Quantum chromodynamics, Chiral symmetry, Quark models, heavy-light mesons, meson-baryon interactions

PACS: 12.38.-t, 14.40.Lb, 12.39.Pn, 11.30.Rd, 13.75.Jz

\section{INTRODUCTION}

The low-energy scattering of strange and charmed mesons by nucleons has been subject of many investigations during the last years $[1,2,3,4]$. Most of the studies have concentrated attention on two basic approaches, meson-exchange models and constituent quark models. In Ref. [1], a meson-exchange model was developed as an extension of the Bonn model for the NN interaction to study the $K^{+} N$ reaction. This work considered contributions of vector $(\rho, \omega)$ and scalar $(\sigma)$ mesons together with higher-order diagrams involving $N, \Delta, K$, and $K^{*}$ intermediate states. A good description of phase shifts and cross sections was obtained, but an additional phenomenological short-range repulsive contribution of a $\sigma_{\text {rep }}$ was required to explain simultaneously phase shifts for $S$ and $P$ waves. This model was sophisticated in Ref. [4], where the authors replace the unphysical $\sigma_{\text {rep }}$ contribution by quark-gluon exchange. The result was a satisfactory description of the $S$ as well as higher partial waves. Recently [2, 3] SU(4) flavor symmetry was invoked to extend this hybrid model to the $\bar{D} N$ system $\left(\bar{D}\right.$ stands $\bar{D}^{0}$ and $\left.D^{-}\right)$. In this study it was shown that quark-gluon interchange contributes with $50 \%$ to the $S$-wave phase shifts and $\sigma, \omega$ and $\rho$ meson exchanges contribute with other $50 \%$.

In spite of the many successes achieved by constituent quark models in the description of low energy hadronic spectra and interactions, they are clearly unable to explain the source of the constituent quark masses which are supposed to be generated by dynamical chiral symmetry breaking $(\mathrm{D} \chi \mathrm{SB})$. In view of this, such a model is clearly limited for studying chiral effects in a medium at finite baryon density and finite temperature. With these facts in mind, we apply here the approach used in the Refs $[2,3]$ to study the

CP1296, XI Hadron Physics

edited by M. Nielsen, F. S. Navarra, and M. E. Bracco

(C) 2010 American Institute of Physics 978-0-7354-0848-7/10/\$30.00 
$K N$ and $\bar{D} N$ interaction, but now with a quark model inspired in the QCD Hamiltonian in Coulomb gauge [5]. The model is based on a field theoretic Hamiltonian that confines color and realizes $\mathrm{D} \chi \mathrm{SB}[6]$. The model allows to obtain effective meson-baryon potentials that can be used in a Lippmann-Schwinger equation to calculate phase shifts. In order to compare with the results obtained in Ref. [2], we add contributions from one-meson exchanges.

\section{QUARK MODEL WITH DYNAMICAL CHIRAL SYMMETRY BREAKING}

Our starting point is a microscopic Hamiltonian inspired in Coulomb gauge QCD [6]

$$
\begin{aligned}
H & =\int d \mathbf{x} \Psi^{\dagger}(\vec{x})\left[-i \vec{\alpha} \cdot \vec{\nabla}+\beta m_{0}\right] \Psi(\vec{x})-\frac{1}{2} \int d \mathbf{x} d \mathbf{y} \rho^{a}(\vec{x}) V_{C}(|\vec{x}-\vec{y}|) \rho^{a}(\vec{y}) \\
& +\frac{1}{2} \int d \mathbf{x} d \mathbf{y} J_{i}^{a}(\vec{x}) V_{i j}(|\vec{x}-\vec{y}|) J_{j}^{a}(\vec{y}),
\end{aligned}
$$

where $\Psi(\vec{x})$ is the quark field operator, $m_{0}$ is the current-quark mass matrix, and $\rho^{a}(\vec{x})$ and $J_{i}^{a}(\vec{x})$ are the color charge and current densities given by

$$
\rho^{a}(\vec{x})=\Psi^{\dagger}(\vec{x}) \frac{\lambda^{a}}{2} \Psi(\vec{x}), \quad J_{i}^{a}(\vec{x})=\Psi^{\dagger}(\vec{x}) \frac{\lambda^{a}}{2} \alpha_{i} \Psi(\vec{x}) .
$$

In Eq. (1), $V_{C}(|\vec{x}-\vec{y}|)$ is the Coulomb potential, and $V_{i j}(|\vec{x}-\vec{y}|)$ is a transverse-gluon hyperfine potential

$$
V_{i j}(|\vec{x}-\vec{y}|)=\left(\delta_{i j}-\frac{\nabla_{i} \nabla_{j}}{\nabla_{j}}\right) V_{T}(|\vec{x}-\vec{y}|) .
$$

The quark field operator is expanded in a basis of plane-wave spinors $u$ and $v$ as

$$
\Psi(\vec{x})=\int \frac{d \mathbf{x}}{(2 \pi)^{3 / 2}} \sum_{s= \pm 1 / 2}\left[u_{s}(\vec{k}) q_{s}(\vec{k})+v_{s}(\vec{k}) \bar{q}_{s}^{\dagger}(-\vec{k})\right] e^{i \vec{k} \cdot \vec{r}}
$$

where

$$
u_{s}(\vec{k})=\sqrt{\frac{E_{k}+M_{k}}{2 E_{k}}}\left(\begin{array}{c}
1 \\
\frac{\vec{\sigma} \cdot \vec{k}}{E_{k}+M_{k}}
\end{array}\right) \chi_{s}, \quad v_{s}(\vec{k})=\sqrt{\frac{E_{k}+M_{k}}{2 E_{k}}}\left(\begin{array}{c}
-\frac{\vec{\sigma} \cdot \vec{k}}{E_{k}+M_{k}} \\
1
\end{array}\right) \chi_{s}^{c},
$$

where $M_{k}$ is the constituent-quark mass function. The mass function is obtained by solving a Schwinger-Dyson equation for the quark propagator, which results in

$$
M_{k}=m_{0}+\frac{2}{3} \int \frac{d \mathbf{q}}{(2 \pi)^{3} E_{q}}\left[f_{1}(\vec{k}, \vec{q}) V_{C}(|\vec{k}-\vec{q}|)+g_{1}(\vec{k}, \vec{q}) V_{T}(|\vec{k}-\vec{q}|)\right] .
$$

Once the potentials $V_{C}(|\vec{x}-\vec{y}|)$ and $V_{T}(|\vec{x}-\vec{y}|)$ are specified, this integral equation can be solved by iteration for different current quark masses $m_{0}$. 


\section{COULOMB GAUGE QUARK-MODEL MESON-BARYON INTERACTION}

Having obtained the mass function, one proceeds to the derivation of an effective mesonbaryon potential. For this, one needs hadron wave functions. They are obtained variationally using an approximation scheme that allows to perform almost all calculations analytically. Specifically, we retain terms up to $O\left(k^{2} / M^{2}\right)$ in the expansion of the spinors $u_{s}(k)$ and $v_{s}(k)$ to obtain a Fermi-Breit type of Hamiltonian. The dominant components of the $q q$ and $q \bar{q}$ interactions can be written in momentum space as

$$
V(\vec{q})=V_{C}(\vec{q})+\frac{2}{3} \frac{q^{2}}{M_{1} M_{2}} \vec{S}_{1} \cdot \vec{S}_{2} V_{T}(\vec{q}),
$$

where $V_{C}(q)$ and $V_{T}(q)$ are the Fourier transforms of $V_{C}(|\vec{x}|)$ and $V_{T}(|\vec{x}|)$. The part of the Hamiltonian relevant for our study can be written in a compact notation as

$$
\begin{aligned}
H_{q \bar{q}} & =T(\mu) q_{\mu}^{\dagger} q_{\mu}+T(v) \bar{q}_{\nu}^{\dagger} \bar{q}_{v}+\frac{1}{2} V_{q q}(\mu v ; \sigma \rho) q_{\mu}^{\dagger} q_{v}^{\dagger} q_{\rho} q_{\sigma} \\
& +\frac{1}{2} V_{\bar{q} \bar{q}}(\mu \nu ; \sigma \rho) \bar{q}_{\mu}^{\dagger} \bar{q}_{\nu}^{\dagger} \bar{q}_{\rho} \bar{q}_{\sigma}+V_{q \bar{q}}(\mu \nu ; \sigma \rho) q_{\mu}^{\dagger} \bar{q}_{\nu}^{\dagger} \bar{q}_{\rho} q_{\sigma},
\end{aligned}
$$

where $\mu, v, \cdots$ represent the set of all quantum numbers of quarks (momentum, color, spin and flavor). From this quark Hamiltonian one can obtain an effective meson-baryon potential using e.g. the Fock-Tani representation. Given baryon $\Psi$ and meson $\Phi$ wave functions (derived for the same quark Hamiltonian), the meson-baryon potential is

$$
\begin{aligned}
V(a b, c d)= & -3 \phi_{c}^{* \mu v_{1}} \psi_{d}^{* v \mu_{2} \mu_{3}} V_{q q}(\mu v ; \sigma \rho) \phi_{a}^{\rho v_{1}} \psi_{b}^{\sigma \mu_{2} \mu_{3}} \\
& -3 \phi_{c}^{* \sigma \rho} \psi_{d}^{* \mu_{1} \mu_{2} \mu_{3}} V_{q \bar{q}}(\mu v ; \sigma \rho) \phi_{a}^{\mu_{1} \rho} \psi_{b}^{\mu \mu_{2} \mu_{3}} \\
& -6 \phi_{c}^{* \mu_{1} v_{1}} \psi_{d}^{* v \mu \mu_{3}} V_{q q}(\mu v ; \sigma \rho) \phi_{a}^{\rho v_{1}} \psi_{b}^{\mu_{1} \sigma \mu_{3}} \\
& -6 \phi_{c}^{* v_{1} v} \psi_{d}^{* v_{1} \mu \mu_{3}} V_{q \bar{q}}(\mu v ; \sigma \rho) \phi_{a}^{v_{1} \rho} \psi_{b}^{\mu_{1} \sigma \mu_{3}},
\end{aligned}
$$

where $a, b, \cdots$ represent the set of all hadron quantum numbers. The explicit forms the $V_{C}$ and $V_{T}$ of the microscopic Hamiltonian necessary in the calculations are given in [7].

\section{MESON-EXCHANGE CONTRIBUTIONS}

The interacting Lagrangian densities we use are given in Refs. [2] and [8]. The (OBE) potentials derived from these Lagrangian densities lead to the following expressions for the vector-meson $(v=\rho, \omega)$ and scalar-meson $(\sigma)$ exchanges,

$$
\begin{gathered}
V^{v}\left(\mathbf{p}^{\prime}, \mathbf{p}\right)=\frac{g_{N N v} g_{P P v}}{\sqrt{(2 \pi)^{6} 4 \omega(p) \omega\left(p^{\prime}\right)}}\left(p^{\prime}+p\right)_{\mu} \Delta_{v}^{\mu v}(q) \\
\times \quad\left[\bar{u}\left(\mathbf{p}^{\prime}, s^{\prime}\right) \gamma_{\mu} u(\mathbf{p}, s)+\left(\frac{\kappa_{v}}{2 m_{N}}\right) \bar{u}\left(\mathbf{p}^{\prime}, s^{\prime}\right) i \sigma_{\mu v} q^{v} u(\mathbf{p}, s)\right], \\
V^{\sigma}\left(\mathbf{p}^{\prime}, \mathbf{p}\right)=\frac{g_{N N \sigma} g_{P P \sigma}}{\sqrt{(2 \pi)^{6} 4 \omega(p) \omega\left(p^{\prime}\right)}} \Delta_{\sigma}(q) \bar{u}\left(\mathbf{p}^{\prime}, s^{\prime}\right) u(\mathbf{p}, s) .
\end{gathered}
$$




\section{NUMERICAL RESULTS}

In Figs. 1-2 we present our numerical results for the $D N$ and $K N S$-wave phase-shifts for the $I=0$ and $I=1$ isospin channels.
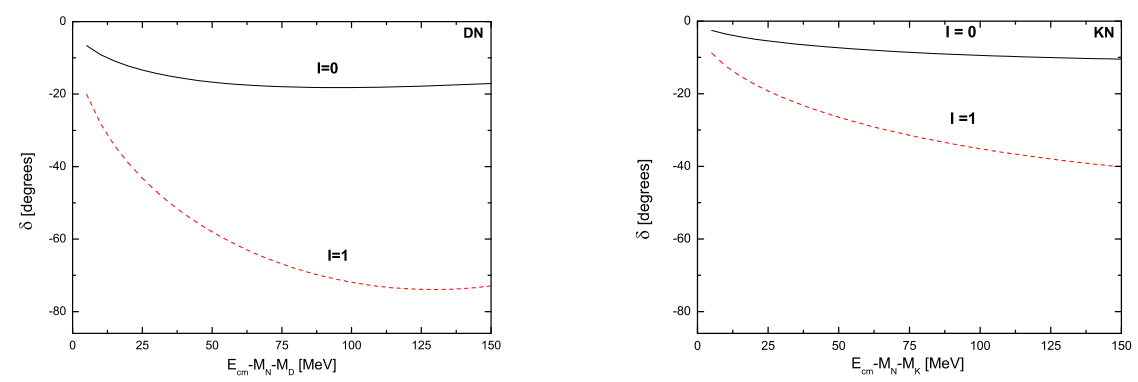

FIGURE 1. Quark interchange $\mathrm{S}$-wave phase-shift for isospin $\mathrm{I}=0$ and $\mathrm{I}=1$.
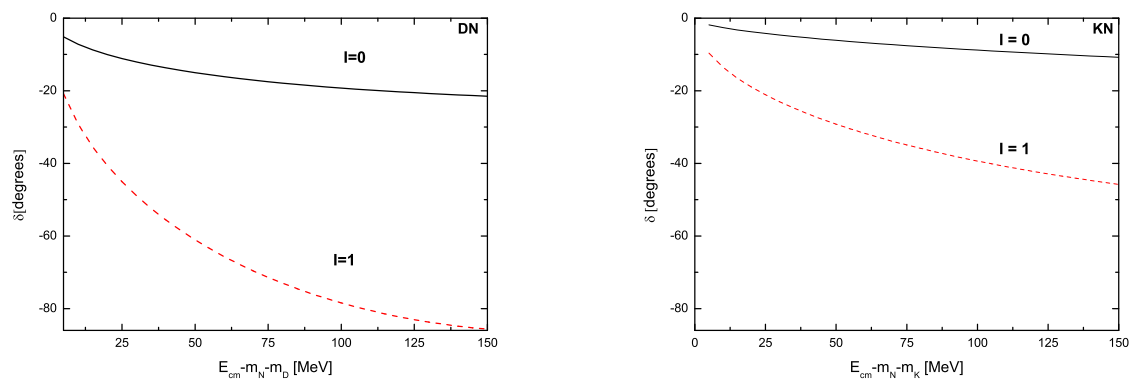

FIGURE 2. Quark interchange and Meson-exchange S-wave phase-shift for isospin $I=0$ and $I=1$.

\section{ACKNOWLEDGMENTS}

The author thanks the guidance of G. Krein. Work financed by CNPq (Brazilian agency).

\section{REFERENCES}

1. R. Buettgen et al.,Nucl. Phys. A506, 586 (1990).

2. J. Haidenbauer, G. Krein, U. -G. Meißner and A. Sibirtsev, Eur. Phys. J. A33, 107 (2007).

3. J. Haidenbauer, G. Krein, U. G. Meissner and A. Sibirtsev, Eur. Phys. J. A 37, 55 (2008).

4. D. Hadjimichef, J. Haidenbauer and G. Krein, Phys. Rev. C66, 055214 (2002).

5. A. P. Szczepaniak and E. S. Swanson, Phys. Rev. Lett. 87, 072001 (2001).

6. F. J. Llanes-Estrada,S. R.Cotanch,A. P. Szcepaniak and E. S.Swanson, Phys. Rev.C70, 035202 (2004).

7. G. Krein and V. E. Vizcarra, PoS CONFINEMENT8 100 (2008).

8. Z. -w. Lin and C. M. Ko, Phys. Rev. C62, 034903 (2000). 
Copyright of AIP Conference Proceedings is the property of American Institute of Physics and its content may not be copied or emailed to multiple sites or posted to a listserv without the copyright holder's express written permission. However, users may print, download, or email articles for individual use. 